\title{
COMPORTAMENTO FENOLÓGICO, NUTRICIONAL E DE PRODUTIVIDADE DE CULTIVARES DE SOJA NO SUDESTE PARAENSE
}

\author{
Marcele de Cássia Henriques dos Santos Moraes ${ }^{1}$ Letícia Cunha da Hungria², Jamil Chaar El-Husny ${ }^{3}$, \\ Priscila Pereira Sacramento ${ }^{4}$, Izabelle Pereira Andrade 5
}

\begin{abstract}
RESUMO - Avaliar o potencial de diferentes cultivares de soja em condições complexas como da região amazônica é necessário para investigar a capacidade de adaptação e o comportamento em vários aspectos dessas cultivares. Neste sentido, objetivou-se avaliar o comportamento fenológico, nutricional e de produtividade de cultivares de soja em condições edafoclimáticas do sudeste Paraense. Para isso, foram testadas nove cultivares: BRS 279 RR, BRS 333 RR, BRS 325 RR, BRS 9090 RR, BRS 8990 RR, BRS Sambaíba RR, P98R31, P98Y51 e P98Y70. O delineamento experimental foi em blocos casualizados, com densidade de 240.000 plantas ha ${ }^{-1}$, com quatro repetições. Foram avaliadas altura de planta e de inserção do primeiro legume $(\mathrm{cm})$, floração e maturação (dias) e produtividade de grãos $\left(\mathrm{kg} \mathrm{ha}^{-1}\right)$. Além disso, foi quantificado o teor de $\mathrm{N}, \mathrm{P}$ e $\mathrm{K}$ nas folhas. Para altura de plantas, destacaram-se as cultivares BRS 9090 RR, BRS 333 RR e P98Y70, com alturas de 73,25; 72,25 e 71 cm, respectivamente. A inserção do primeiro legume variou de 8,75 (P98Y51) a 16,75 cm (BRS 333 RR). A floração foi atingida entre 34 (P98R31) e 42 (BRS 333 RR e P98Y70) dias após a emergência. O período de maturação teve início mínimo aos 98 dias (P98R31) e máximo aos 119 dias (BRS 333 RR) após a emergência. As maiores produtividades foram registradas para as BRS's e P98Y70, variando entre 1890 e $3192 \mathrm{~kg} \mathrm{ha}^{-1}$. O teor foliar de N, P, K não diferiu entre as cultivares testadas. A BRS 333 RR, mostrou excelente desempenho das características agronômicas.
\end{abstract}

Palavras chave: características agronômicas, floração, maturação, produtividade de grãos.

\section{PHENOLOGICAL, NUTRITIONAL AND PRODUCTIVITY BEHAVIOR OF SOYBEAN CULTIVARS IN SOUTHEAST OF PARÁ}

\begin{abstract}
Evaluate the potential of different soybean cultivars in complex conditions such as in the Amazon region is necessary to investigate the adaptability and behavior in various aspects of these cultivars. In this sense, the objective was to evaluate the phenological, nutritional and productivity behavior of soybean cultivars under edaphoclimatic conditions in Pará southeast. For this, nine cultivars were tested: BRS 279 RR, BRS 333 RR, BRS 325 RR, BRS 9090 RR, BRS 8990 RR, BRS Sambaiba RR, P98R31, P98Y51 and P98Y70. The experimental design was in randomized blocks, with density of 240.000 plants $\mathrm{ha}^{-1}$, with four replications. Plant height and first pod insertion (cm), flowering and maturation (days) and grain yield $\left(\mathrm{kg} \mathrm{ha}^{-1}\right)$ were evaluated. In addition, was quantified $N, P$ and $K$ leaf contents. For plant height, the cultivars BRS 9090 RR, BRS 333 RR and P98Y70 stood out, with heights of 73.25; 72.25 and $71 \mathrm{~cm}$, respectively. The insertion of the first pod ranged from 8.75 (P98Y51) to $16.75 \mathrm{~cm}$ (BRS 333 RR). Flowering was reached between 34 (P98R31) and 42 (BRS 333RR and P98Y70) days after emergence. The maturation period started at a minimum of 98 days (P98R31) and a maximum of 119 days (BRS 333 RR) after emergence. The highest yields were registered for BRS's and P98Y70, varying between 1890 and $3192 \mathrm{~kg} \mathrm{ha}$. The N, P, K leaf contents did not differ between the cultivars tested. The BRS 333 RR, showed excellent performance of agronomic characteristics.
\end{abstract}

Keywords: agronomic characteristics, amazon conditions, flowering, grain yield, maturation.

\footnotetext{
${ }^{1}$ Mestranda em Produção Agrícola, Universidade Federal Rural de Pernambuco, Garanhuns, PE. E-mail: marcele-moraes@hotmail.com

${ }^{2}$ Doutoranda em Agronomia, Instituto de Ciências Agrárias/UFRA, bolsista CAPES, Belém, PA. Email:leth_hungria@hotmail.com

${ }^{3}$ Pesquisador, Embrapa Amazônia Oriental, Belém, PA. Email: jamil.husny@embrapa.br

${ }^{4}$ Pós-Graduanda em Desenvolvimento Rural Sustentável e Gestão de Empreendimento Agroalimentares, Instituto Federal do Pará, Castanhal, PA. E-mail: priscila.sacrame@gmail.com

${ }^{5}$ Professora, Instituto de Ciências Agrárias/UFRA, Paragominas, PA. Email: izabelle.andrade@ufra.edu.br
} 


\section{INTRODUÇÃO}

A soja (Glycine $\max (\mathrm{L}$.$) Merrill) é uma das$ espécies oleaginosas mais cultivadas no mundo e de grande importância na dieta humana e animal, em função de servir como uma excelente fonte proteica (Bianco et al., 2012). Os grãos de soja são utilizados para consumo in natura ou como matéria-prima básica para a produção de farelo e óleo, dentre outros subprodutos (Mauad et al., 2010; Ferreira et al., 2015).

No Brasil, a soja destaca-se dentre as commodities responsáveis pelos excelentes resultados do agronegócio no país. Isto é resultado, dentre outros fatores, do desenvolvimento de genótipos adaptados às condições de baixa latitude, pelos programas de melhoramento genético, o que provocou a expansão de áreas de cultivo e promoveu alto potencial de produção em diferentes regiões do país, incluindo a região Norte (Peluzio et al., 2010).

A máxima produtividade de grãos, como principal interesse no planejamento da colheita, está estreitamente relacionado ao sistema de produção adotado (Santos et al., 2013), a densidade de semeadura utilizada (Sacramento et al., 2020) e às condições de clima e solo das áreas cultivadas. Os fatores edafoclimáticas, em particular, provocam efeito direto na estatura das plantas, duração do ciclo e produtividade (Amorim et al., 2011; Perini et al., 2012). Portanto, ao escolher determinada cultivar para a semeadura é necessário ter conhecimento do seu desempenho na região elegida.

Para verificar o potencial de diferentes cultivares de soja em condições complexas como da região amazônica é necessário investigar a capacidade de adaptação e o comportamento em vários aspectos dessas cultivares. Neste contexto, o objetivo foi avaliar o comportamento fenológico, nutricional e de produtividade de cultivares de soja em condições edafoclimáticas do sudeste Paraense.

\section{MATERIAL E MÉTODOS}

\section{Descrição da área de estudo}

O estudo foi realizado no ano agrícola 2014/2015, no campo experimental da Embrapa Amazônia Oriental, município de Paragominas ( $02^{\circ} 59^{\prime} 45^{\prime}$ 'S e $47^{\circ} 21^{\prime} 10^{\prime}$ " O), mesorregião do sudeste do estado do Pará, Amazônia brasileira. A área de estudo pertence a uma zona climática tropical chuvosa - Aw (Köppen), com expressivo período de estiagem, temperatura média anual de $26,5^{\circ} \mathrm{C}$, precipitação média anual de $1.800 \mathrm{~mm}$ (Rodrigues et al., 2003). A precipitação média mensal $(\mathrm{mm})$ e temperaturas médias $\left({ }^{\circ} \mathrm{C}\right)$ durante o ano do estudo são apresentadas na Figura 1.

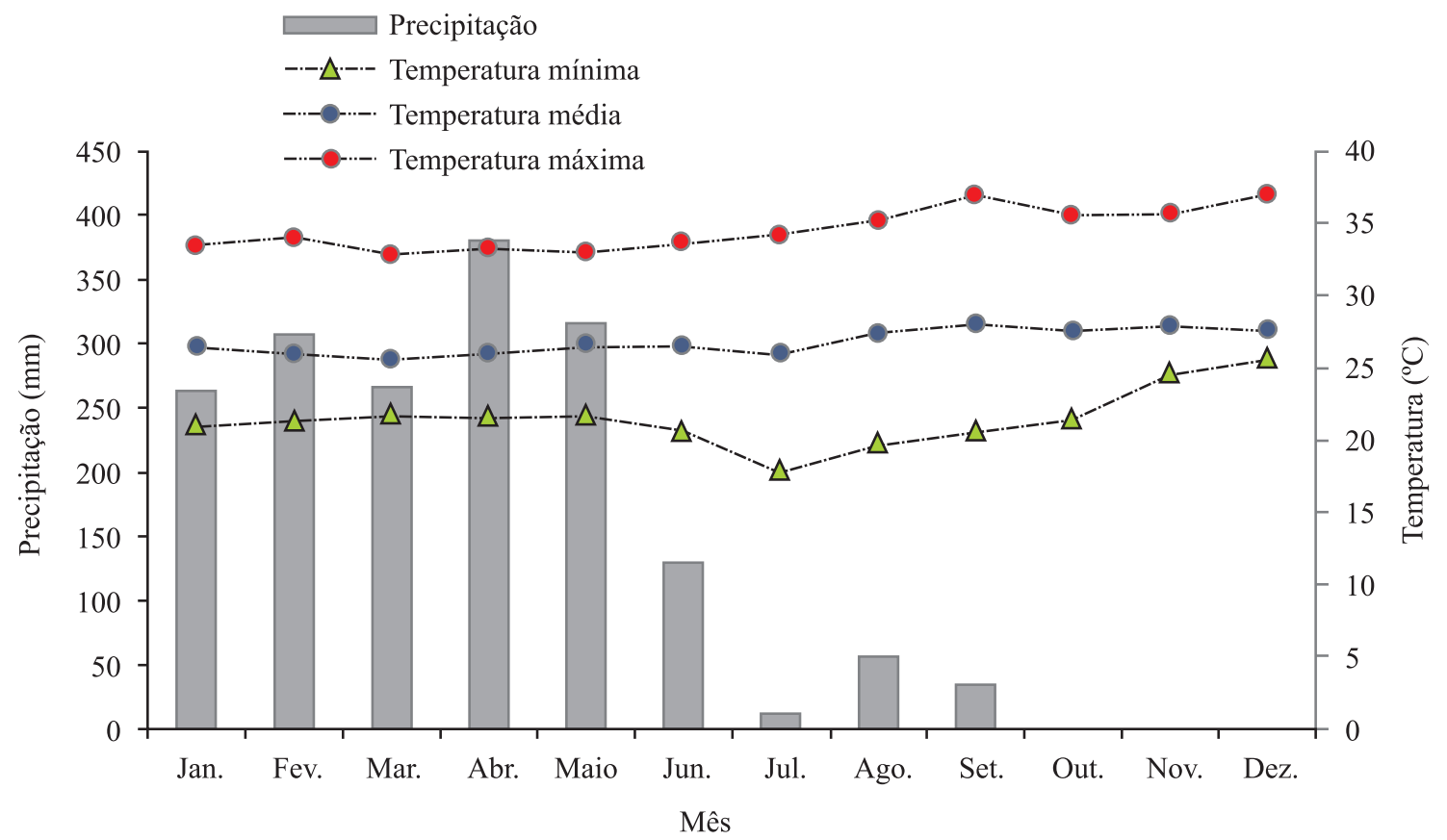

Figura 1 - Precipitação média mensal $(\mathrm{mm})$ e temperaturas mínima, média e máxima $\left({ }^{\circ} \mathrm{C}\right)$ na região de Paragominas - PA, durante o ano do estudo. 
O solo da área é do tipo Latossolo Amarelo, textura argilosa (20 $\mathrm{g} \mathrm{kg}^{-1}$ de areia grossa, $32 \mathrm{~g} \mathrm{~kg}^{-1} \mathrm{de}$ areia fina, $428 \mathrm{~g} \mathrm{~kg}^{-1}$ de silte e $520 \mathrm{~g} \mathrm{~kg}^{-1}$ de argila). As propriedades químicas do solo na camada superficial (0-20 cm) são apresentadas na Tabela 1.

Tabela 1 - Análise química do solo na camada de 0-20 cm, da área experimental, ano agrícola 2014/2015, Paragominas, PA

\begin{tabular}{cccccccccccc}
\hline $\mathrm{pH}$ & $\mathrm{MO}$ & $\mathrm{P}$ & $\mathrm{K}$ & $\mathrm{Na}$ & $\mathrm{Ca}$ & $\mathrm{Ca}+\mathrm{Mg}$ & $\mathrm{Al}$ & $\mathrm{H}+\mathrm{Al}$ & $\mathrm{m} \%$ & $\mathrm{CTC}$ & $\mathrm{V} \%$ \\
\hline $\mathrm{H}_{2} \mathrm{O}$ & $\mathrm{g} / \mathrm{kg}$ & \multicolumn{2}{c}{$-----\mathrm{mg} / \mathrm{dm}^{3}------$} & \multicolumn{3}{c}{$--------\mathrm{cmol}_{\mathrm{c}} / \mathrm{dm}^{3}--------$} & & $\mathrm{cmol}_{\mathrm{c}} / \mathrm{dm}^{3}$ & \\
\hline 5,5 & 28,61 & 6 & 103 & 2 & 2,5 & 3,2 & 0,2 & 3,80 & 5,45 & 3,67 & 47,75 \\
\hline
\end{tabular}

Tratamento e delineamento experimental

$\mathrm{O}$ delineamento experimental foi em blocos completos ao acaso, com nove tratamentos e quatro repetições. Os tratamentos consistiram em nove cultivares de soja com características apresentadas na Tabela 2.

Tabela 2 - Descrição das características das cultivares testadas

\begin{tabular}{lccccc}
\hline Cultivar & $\begin{array}{c}\text { Ciclo* }^{*} \\
\text { (dias) }\end{array}$ & $\begin{array}{c}\text { Densidade } \\
\left(\text { mil plantas ha }{ }^{-1}\right.\end{array}$ & GM & \multicolumn{2}{c}{ Hábito de } \\
crescimento & $\begin{array}{c}\text { Altura média de } \\
\text { planta }\end{array}$ \\
\hline P98Y70 & $115-120$ & $180-220$ & 8.7 & Determinado & $57 \mathrm{~cm}$ \\
P98Y51 & $111-123$ & $220-350$ & 8.5 & Determinado & $44 \mathrm{~cm}$ \\
P98R31 & $115-120$ & $220-350$ & 8.3 & Determinado & $73 \mathrm{~cm}$ \\
BRS Sambaíba RR & $104-131$ & $150-250$ & 9.3 & Determinado & $76 \mathrm{~cm}$ \\
BRS 8990RR & $112-120$ & $200-230$ & 8.9 & Determinado & $74 \mathrm{~cm}$ \\
BRS 9090RR & $100-127$ & $180-230$ & 9.0 & Determinado & $83 \mathrm{~cm}$ \\
BRS 325RR & $118-121$ & $200-230$ & 9.0 & Determinado & $69 \mathrm{~cm}$ \\
BRS 333RR & $118-128$ & $180-220$ & 9.4 & Determinado & $76 \mathrm{~cm}$ \\
BRS 279RR & $108-114$ & $220-250$ & 8.8 & Determinado & $65 \mathrm{~cm}$ \\
\hline
\end{tabular}

*Ciclo de maturação; GM: grupo de maturação.

Fonte: VILELA et al. (2016).

As parcelas experimentais possuíam dimensões de 2 x $5 \mathrm{~m}$, com área útil de $4 \mathrm{~m}^{2}$ e espaçamento de $0,5 \mathrm{~m}$ entre linhas. Duas linhas de plantas úteis foram utilizadas para avaliar a produtividade de grãos na colheita, descontando $0,5 \mathrm{~m}$ de cada extremidade, sendo as demais utilizadas para outras avaliações ou constituindo as bordas. A densidade da semeadura para todas as cultivares foi de 240.000 plantas ha' ${ }^{-1}$.

\section{Semeadura e manutenção em campo}

Todas as parcelas receberam adubação em função dos resultados da análise química do solo (Tabela 1) e da produtividade da cultivar, estimada para $2.500 \mathrm{~kg}$ $\mathrm{ha}^{-1}$. Aplicou-se o equivalente a $300 \mathrm{~kg} \mathrm{ha}^{-1}$ da fórmula fertilizante 08-40-10 (NPK), correspondendo a $8 \mathrm{~kg}$ de N, $40 \mathrm{~kg}$ de $\mathrm{P}_{2} \mathrm{O}_{5}$ e $10 \mathrm{~kg}$ de $\mathrm{K}_{2} \mathrm{O}$, com adubação realizada em fundação.

As sementes foram tratadas com $200 \mathrm{~mL}$ de fungicida Standak Top (Piraclostrobin + tiofanato de metila + Fipronil) por $100 \mathrm{~kg}$ de sementes, para proteger as plântulas contra o ataque de pragas, e fungos de sementes no período inicial de desenvolvimento da cultura, conforme recomendações do fabricante.

\section{Características avaliadas}

Foram registradas as seguintes variáveis conforme metodologia de Rocha et al. (2009): 
Número de dias para a floração: consideraram-se os dias entre a emergência até o início do florescimento, estádio fenologicamente definido quando $50 \%$ das plantas da parcela útil possuíam pelo menos uma flor aberta.

Número de dias para a maturação: foi observado o intervalo determinado em dias após a emergência até a maturação, estágio fenologicamente definido pela presença de $50 \%$ das plantas da área útil, considerando $95 \%$ dos legumes maduros.

Altura de plantas: distância ( $\mathrm{cm})$, entre a superfície do solo e o ápice da haste principal de 10 plantas tomadas aleatoriamente, quando estas se encontravam no estágio reprodutivo $\mathrm{R} 8$, em que $95 \%$ dos legumes atingiram a cor de legume maduro, variando com cada cultivar.

Altura de inserção do primeiro legume: distância (cm) entre a superfície do solo e a inserção do primeiro legume.

Produtividade: avaliada na maturidade final, após a colheita através de debulha manual dos legumes e pesagem dos grãos colhidos na área útil de cada parcela, os dados obtidos (gramas por parcelas) foram transformados para $\mathrm{kg} \mathrm{ha}^{-1}$, com produtividade corrigida para teor de umidade de 13\% (Freitas, 2011; Cavalcante et al., 2014). Conforme a fórmula:

$$
P F=\frac{P I \times(100=U I)}{(100-U F)}
$$

Em que:

PF: peso final da amostra (peso corrigido);

PI: peso inicial da amostra;

UI: umidade inicial da amostra, em percentagem;

UF: umidade final da amostra (13\%).

\section{Teor de nutrientes na planta}

As mesmas plantas avaliadas quanto à altura foram cortadas próximas ao solo, as folhas foram lavadas com água destilada e levadas a estufa a $65{ }^{\circ} \mathrm{C}$ com circulação forçada de ar até atingirem massa constante para obtenção da massa seca. Após serem secas, as folhas foram moídas e digeridas em solução nitro-perclórica 2:1 para determinação de $\mathrm{P}$ e $\mathrm{K}$, e solução sulfúrica para determinação de $\mathrm{N}$ de acordo com a metodologia de Malavolta et al. (1997). O N foi determinado por destilação Kjeldahl; o P por colorimetria do metavanadato e o K por fotometria de chama (EMBRAPA, 2011).

\section{Análise de dados}

Os dados coletados foram submetidos à análise de variância (ANOVA) para comparar as características estudadas entre as cultivares de soja. Quando significativas, as médias foram comparadas pelo teste de Scott Knott $(\mathrm{p}<0,05)$ por meio do software SISVAR, versão 5.6 (Ferreira, 2014).

\section{RESULTADOS E DISCUSSÃO}

Os resultados da análise de variância demonstraram que houve diferença $(p<0,05)$ entre os genótipos, em suas interações com o ambiente, para todos os caracteres avaliados, exceto para nutrição da planta (Tabela 3), permitindo inferir sobre a influência da variabilidade genética das cultivares consideradas no estudo.

Tabela 3 - Análise de variância para altura de planta (ALT) e de inserção do primeiro legume (IPL), floração (FLOR), maturação (MAT), produtividade (PROD) e teores médios foliares de nitrogênio $(\mathrm{N})$, fósforo $(\mathrm{P})$ e potássio $(\mathrm{K})$ de nove cultivares de soja, na safra 2014/2015. Paragominas, PA

\begin{tabular}{lccccccccc}
\hline & \multicolumn{10}{c}{ Quadrado médio } \\
\cline { 2 - 9 } Fonte de variação & GL & $\begin{array}{c}\text { ALT } \\
(\mathrm{cm})\end{array}$ & $\begin{array}{c}\text { IPL } \\
(\mathrm{cm})\end{array}$ & $\begin{array}{c}\text { FLOR } \\
(\text { dias })\end{array}$ & $\begin{array}{c}\text { MAT } \\
(\text { dias })\end{array}$ & $\begin{array}{c}\text { PROD } \\
\left(\mathrm{kg} \mathrm{ha}^{-1}\right)\end{array}$ & $\begin{array}{c}\mathrm{N} \\
\left(\mathrm{g} \mathrm{kg}^{-1}\right)\end{array}$ & $\begin{array}{c}\mathrm{P} \\
\left(\mathrm{g} \mathrm{kg}^{-1}\right)\end{array}$ & $\begin{array}{c}\mathrm{K} \\
\left(\mathrm{g} \mathrm{kg}^{-1}\right)\end{array}$ \\
\hline Cultivares & 8 & $661,13^{*}$ & $23,50^{*}$ & $24,52^{*}$ & $119,68^{*}$ & $809.438,25^{*}$ & $11,57^{\mathrm{ns}}$ & $0,61^{\mathrm{ns}}$ & $95,41^{\mathrm{ns}}$ \\
Blocos & 3 & $268,25^{\text {ns }}$ & $5,06^{\text {ns }}$ & $4,29^{\text {ns }}$ & $0,54^{\text {ns }}$ & $191.828,10^{\mathrm{ns}}$ & $11,16^{\mathrm{ns}}$ & $0,05^{\mathrm{ns}}$ & $165,92^{\mathrm{ns}}$ \\
Resíduo & 24 & 43,60 & 2,39 & 1,37 & 1,73 & 7668,18 & 10,73 & 0,42 & 233,64 \\
CV $(\%)$ & & 11,17 & 12,15 & 3,03 & 1,24 & 10,48 & 8,06 & 22,21 & 34,66 \\
\hline
\end{tabular}

ns: não significativo; * : significativo a $5 \%$ de probabilidade pelo teste $\mathrm{F}$. 
As alturas de plantas variaram entre 38 a $73 \mathrm{~cm}$, com as maiores médias alcançadas pelas cultivares P98Y70, BRS 9090 RR e BRS 333 RR e incremento de 13\% na altura média das plantas quando comparadas as cultivares BRS 279 RR, BRS 8990 RR e BRS Sambaíba RR, que não diferiram entre si (Tabela 4). Estaturas inferiores foram encontradas para as cultivares P98R31, BRS 325 RR e P98Y51.

Tabela 4 - Altura de plantas e de inserção do primeiro legume (cm) de nove cultivares de soja cultivadas em condições edafoclimáticas do sudeste paraense, safra 2014/2015

\begin{tabular}{lcc}
\hline & \multicolumn{2}{c}{ Altura } \\
\cline { 2 - 3 } Cultivares & $\begin{array}{c}\text { Plantas } \\
(\mathrm{cm})\end{array}$ & $\begin{array}{c}\text { Inserção do } \\
\text { primeiro legume } \\
(\mathrm{cm})\end{array}$ \\
\hline P98Y70 & $71,00 \mathrm{a}$ & $14,00 \mathrm{~b}$ \\
BRS 279 RR & $62,75 \mathrm{~b}$ & $13,25 \mathrm{~b}$ \\
BRS 9090 RR & $73,25 \mathrm{a}$ & $13,75 \mathrm{~b}$ \\
P98R31 & $38,00 \mathrm{c}$ & $10,50 \mathrm{c}$ \\
BRS8990 RR & $61,00 \mathrm{~b}$ & $14,25 \mathrm{~b}$ \\
BRS 333 RR & $72,25 \mathrm{a}$ & $16,75 \mathrm{a}$ \\
BRS 325 RR & $49,75 \mathrm{c}$ & $10,50 \mathrm{c}$ \\
P98Y51 & $42,25 \mathrm{c}$ & $8,75 \mathrm{c}$ \\
BRS Sambaíba RR & $64,75 \mathrm{~b}$ & $13,00 \mathrm{~b}$ \\
Média & 59,44 & 12,75 \\
\hline
\end{tabular}

*Médias seguidas por letras distintas nas colunas diferem entre si pelo teste de Scott-Knott $(\mathrm{p}<0,05)$.

A altura de plantas é uma característica fitotécnica fundamental e altamente influenciável pelo genótipo (Peluzio, 2006), podendo variar de 20 a $150 \mathrm{~cm}$ ou mais conforme a cultivar e o local de cultivo (Sediyama et al., 1985; Rocha, 2009). No entanto, o mais indicado é que as plantas de soja alcancem uma altura acima de $60 \mathrm{~cm}$ para facilitar a colheita mecanizada, por ocasião da maturação e reduzir as perdas de grãos na operação (Braz et al., 2010; Rossi, 2012). Isto sugere que, dentre as nove cultivares testadas, cinco estão dentro da faixa ideal de altura, com exceção das cultivares P98R31, BRS 325 RR e P98Y51.

Em estudo do comportamento de três cultivares de soja, Peluzio et al. (2010), cultivando-as em diferentes épocas, na condições da região Centro-Sul do estado do Tocantins, registrou, para a cultivar P98Y70, alturas médias de 68,2; 74,6 e 79,2 cm sob densidades de 6, 10 e 14 plantas por metro linear, respectivamente. Sacramento et al. (2020), ao avaliarem o potencial produtivo das cultivares BRS 8990 RR e BRS 9090 RR, em condições edafoclimáticas próximas deste estudo, encontraram alturas médias de 57,0 e $62,7 \mathrm{~cm}$, respectivamente, quando utilizadas 250.000 plantas ha ${ }^{-1}$.

Em relação à altura de inserção do primeiro legume houve variação na faixa de $8,75 \mathrm{~cm}$ a $16,75 \mathrm{~cm}$, com maior altura média observada para a cultivar BRS 333 RR (Tabela 4). Esta variável aliada à altura de plantas são características intrínsecas a genética, mas que podem sofrer influências de vários fatores edafoclimáticos (Costa, 2013). No entanto, o destaque da cultivar BRS 333 RR em ambas as alturas (planta e inserção do primeiro legume) comprova, de certa forma, a relação existente entre estas variáveis, justificada pelo alto grau de correlação $\left(\mathrm{r}=0,73^{*}\right)$ (Tabela 7). Desse modo é fundamental o conhecimento da contribuição da interação genótipo $\mathrm{x}$ ambiente no fenótipo da cultivar (PIRES et al., 2012). Comportamento semelhante foi observado para as cultivares P98R31, BRS 325 RR e P98Y51, que apresentaram as menores alturas de planta e de inserção do primeiro legume.

Somado a isso, quando o plantio é realizado em época não apropriada, a altura da planta é reduzida e há tendência de desenvolvimento dos legumes muito próximos ao solo. A inserção do primeiro legume deve ser observada no mínimo de 10 a $12 \mathrm{~cm}$ em solos de topografia plana e aproximadamente $15 \mathrm{~cm}$ em terrenos mais inclinados (Sediyama et al., 1985; Amorim et al., 2011). Entre as cultivares estudadas somente a cultivar P98Y51 não apresentou altura de inserção do primeiro legume adequado.

A variação entre os genótipos testados com relação à duração do período entre a emergência e o florescimento e entre a emergência e a maturação foi de 42 e 119 dias para a BRS 333 RR e de 34 e 98 dias para a P98R31, que apresentaram os maiores e menores ciclos, respectivamente (Tabela 5). O estudo dessas variáveis é importante para o estabelecimento da época de semeadura, onde algumas cultivares, dependendo da indicação de cada região, pode ser semeada precocemente ou tardiamente.

As cultivares podem ser classificadas por grupo de maturação, com o objetivo de facilitar a tomada de decisões dos usuários quanto às épocas de semeadura, à diversidade de ciclos das cultivares nas propriedades e aos sistemas de sucessão/rotação com outras culturas. A cultivar P98R31 pode ser classificada como uma das cultivares mais precoce, com médias para dias de maturação inferiores das demais cultivares, enquanto a BRS 333 RR foi a mais tardia. As épocas de semeadura, em interação com o ciclo das 
cultivares, podem ainda determinar diferenças significativas sobre a quantidade de grãos produzidos $\left(\mathrm{r}=0,56^{*}\right)$ (Tabela 7$)$.

Tabela 5 - Floração e maturação (dias) em nove cultivares de soja cultivadas em condições edafoclimáticas do sudeste paraense, na safra 2014/2015

\begin{tabular}{lcc}
\hline Cultivares & Floração (dias) & Maturação (dias) \\
\hline P98Y70 & $42 \mathrm{~d}$ & $110 \mathrm{c}$ \\
BRS 279 RR & $38 \mathrm{~b}$ & $105 \mathrm{~b}$ \\
BRS 9090 RR & $40 \mathrm{c}$ & $105 \mathrm{~b}$ \\
P98R31 & $34 \mathrm{a}$ & $98 \mathrm{a}$ \\
BRS 8990 RR & $39 \mathrm{c}$ & $106 \mathrm{~b}$ \\
BRS 333 RR & $42 \mathrm{~d}$ & $119 \mathrm{~d}$ \\
BRS 325 RR & $38 \mathrm{~b}$ & $106 \mathrm{~b}$ \\
P98Y51 & $37 \mathrm{~b}$ & $105 \mathrm{~b}$ \\
BRS Sambaíba RR & $40 \mathrm{c}$ & $106 \mathrm{~b}$ \\
Média & 39 & 107 \\
\hline
\end{tabular}

*Médias seguidas por letras distintas nas colunas diferem entre si pelo teste de Scott-Knott $(\mathrm{p}<0,05)$.

Quanto à produtividade de grãos, houve variação entre 1.890 a $3.192 \mathrm{~kg} \mathrm{ha}^{-1}$ (Figura 2). Das nove cultivares testadas, cinco (P98Y70, BRS 333 RR, BRS 9090 RR, BRS 8990 RR e BRS 279 RR) alcançaram rendimentos de grãos mais elevados e dentro do mesmo patamar de produtividade (acima de $2.600 \mathrm{~kg} \mathrm{ha}^{-1}$ ), com incremento de $47,2 \%$ quando comparados aos rendimentos obtidos com as cultivares P98Y31 e P98Y51.

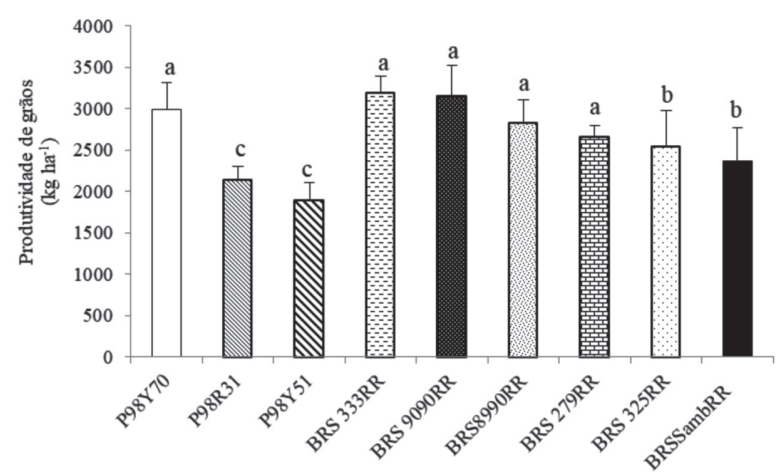

Figura 2 - Produtividade de grãos $\left(\mathrm{kg} \mathrm{ha}^{-1}\right)$ de nove cultivares comerciais de soja em condições edafoclimáticas do sudeste paraense.

*Médias seguidas por letras distintas nas colunas diferem entre si pelo teste de Scott-Knott $(\mathrm{p}<0,05)$.
Os valores de produtividade encontrados neste estudo foram inferiores aos de Sacramento et al. (2020), que avaliando as cultivares BRS 8990 RR e BRS 9090 RR, no sudeste do Pará e densidade de 250.000 plantas $\mathrm{ha}^{-1}$, registraram médias de 3306 e $3262 \mathrm{~kg} \mathrm{ha}^{-1}$ para os materiais testados, respectivamente. Por outro lado, os rendimentos das cultivares BRS 333 RR e BRS 8990 RR foram maiores $16 \%$ e $23 \%$, neste estudo, quando comparados aos valores médios encontrados por Borges et al. (2012), em Belterra-PA.

Os teores foliares de nitrogênio $(\mathrm{N})$, fósforo $(\mathrm{P})$ e potássio $(\mathrm{K})$ não diferiram entre as nove cultivares testadas (Tabela 6). Diferentes genótipos possuem capacidades distintas de aquisição de nutrientes do solo, uma vez que genótipos mais eficientes, por meio de alterações morfológicas e/ou fisiológicas podem aumentar a absorção de nutrientes (Römheld \& Kirkby, 2010). Isto sugere que, mesmo havendo diferença genética entre as cultivares, a eficiência dos mecanismos de absorção ou translocação destes nutrientes são semelhantes entre os genótipos.

Tabela 6 - Resultados médios dos teores foliares de nitrogênio $(\mathrm{N})$, fósforo $(\mathrm{P})$ e potássio $(\mathrm{K}) \mathrm{em}$ nove cultivares de soja, na safra 2014/2015

\begin{tabular}{lccc}
\hline \multirow{2}{*}{ Cultivares } & \multicolumn{3}{c}{ Nutrientes $\left(\mathrm{g} \mathrm{kg}^{-1}\right)$} \\
\cline { 2 - 4 } & $\mathrm{N}$ & $\mathrm{P}$ & $\mathrm{K}$ \\
\hline P98Y70 & $39,22 \mathrm{a}$ & $3,48 \mathrm{a}$ & $46,75 \mathrm{a}$ \\
BRS 279 RR & $43,19 \mathrm{a}$ & $2,59 \mathrm{a}$ & $51,56 \mathrm{a}$ \\
BRS 9090 RR & $41,59 \mathrm{a}$ & $3,09 \mathrm{a}$ & $38,68 \mathrm{a}$ \\
P98R31 & $39,35 \mathrm{a}$ & $2,66 \mathrm{a}$ & $40,18 \mathrm{a}$ \\
BRS 8990 RR & $41,84 \mathrm{a}$ & $3,60 \mathrm{a}$ & $47,74 \mathrm{a}$ \\
BRS 333 RR & $39,79 \mathrm{a}$ & $3,01 \mathrm{a}$ & $45,00 \mathrm{a}$ \\
BRS 325 RR & $38,70 \mathrm{a}$ & $2,61 \mathrm{a}$ & $42,25 \mathrm{a}$ \\
P98Y51 & $42,82 \mathrm{a}$ & $2,51 \mathrm{a}$ & $40,18 \mathrm{a}$ \\
BRS Sambaíba RR & $39,47 \mathrm{a}$ & $3,02 \mathrm{a}$ & $47,90 \mathrm{a}$ \\
Média & 40,66 & 2,95 & 44,10 \\
\hline
\end{tabular}

*Médias seguidas por letras distintas nas colunas diferem entre si pelo teste de Scott-Knott $(\mathrm{p}<0,05)$.

Ressalta-se ainda que, ao considerar o teor de $\mathrm{N}$ adequado para a cultura da soja na faixa de 55 a $58 \mathrm{~g} \mathrm{~kg}^{-1}$ (Malavolta et al., 2006), as plantas de todos os tratamentos encontram-se abaixo da faixa ideal. Por outro lado, no estudo conduzido por Embrapa (1996), estabeleceram, para a cultura da soja, faixas de suficiência de $\mathrm{N}$ entre 40,1 a 
$55,0 \mathrm{~g} \mathrm{~kg}^{-1}$, indicando que somente as plantas provenientes de cinco cultivares (P98Y70, P98R31, BRS 333 RR, BRS 325 RR e BRS Sambaíba RR), entre as nove, não estavam adequadamente nutridas.

O teor médio de $\mathrm{P}$ no tecido foliar dos tratamentos foi de $2,95 \mathrm{~g} \mathrm{~kg}^{-1}$, com todas as cultivares permanecendo dentro da faixa $\left(2,6-5,0 \mathrm{~g} \mathrm{~kg}^{-1}\right)$ adequada para plantas de alto rendimento estabelecida por Embrapa (1996) e todas abaixo quando comparadas a faixa (4,0-5,0 $\left.\mathrm{g} \mathrm{kg}^{-1}\right)$ indicada por Malavolta et al. (2006). Os mesmos autores recomendam que o teor médio de $\mathrm{K}$ na folha deve ser no máximo $25,0 \mathrm{~g} \mathrm{~kg}^{-1}$. Neste estudo, o teor médio de $\mathrm{K}$ no tecido foliar foi de 44,10 $\mathrm{g} \mathrm{kg}^{-1}, 76,4 \%$ acima da quantidade recomendada.

A avaliação do estado nutricional das plantas é uma ferramenta fundamental na identificação dos nutrientes que podem está funcionando como um limitante para o crescimento e produção das culturas, fornecendo informações que possibilitam uma correção mais eficiente (Malavolta, 2006). No entanto, neste estudo, não foi observada relação do desempenho produtivo das cultivares com o estado nutricional (N, P e K) da planta (Tabela 7).

Tabela 7 - Matriz de coeficientes de correlação de Pearson entre altura de planta (ALT) e de inserção do primeiro legume (IPL), floração (FLOR), maturação (MAT), produtividade (PROD) e teores médios foliares de nitrogênio (N), fósforo (P) e potássio (K) de nove cultivares de soja cultivadas em Paragominas, safra 2014/2015

\begin{tabular}{cccccccc}
\hline Variáveis & IPL & FLOR & MAT & PROD & N & P & K \\
\hline ALT & $0,73^{*}$ & $0,65^{*}$ & $0,59^{*}$ & $0,71^{*}$ & 0,04 & 0,32 & 0,11 \\
IPL & & $0,58^{*}$ & $0,57^{*}$ & $0,76^{*}$ & $-0,06$ & 0,22 & 0,10 \\
FLOR & & $0,73^{*}$ & $0,53^{*}$ & $-0,10$ & 0,32 & 0,05 \\
MAT & & & $0,56^{*}$ & $-0,01$ & 0,13 & 0,05 \\
PROD & & & & $-0,03$ & 0,12 & $-0,01$ \\
N & & & & & 0,15 & $0,38^{*}$ \\
P & & & & & & & 0,30 \\
\hline
\end{tabular}

O comportamento das cultivares mostrou que, mesmo o cultivo sendo conduzido sob condições de solo, aspectos nutricionais e práticas de manejo semelhantes, as variáveis de crescimento, bem como a produtividade variaram em função das características de cada cultivar como o ciclo da planta e o hábito de crescimento.

\section{CONCLUSÕES}

1. Para obter a maior produtividade de grãos nas condições deste estudo no sudeste paraense, as cultivares P98Y70, BRS 333 RR, BRS 9090 RR, BRS 8990 RR e BRS 279 RR são as mais indicadas para o cultivo, com média de $2.964 \mathrm{~kg} \mathrm{ha}^{-1}$.

2. Não houve diferença no estado nutricional $(\mathrm{N}, \mathrm{P}$, e $\mathrm{K})$ do tecido foliar das cultivares, indicando semelhança dos mecanismos de absorção de nutrientes entre os genótipos. Além disso, não houve correlação do estado nutricional com as demais variáveis avaliadas.

\section{LITERATURA CITADA}

AMORIM, F.A.; HAMAWAKI, O.T.; SOUSA, L.B.; LANA, R.M.Q.; HAMAWAKI, C.D.L. Época de semeadura no potencial produtivo de soja em Uberlândia-MG. Semina: Ciências Agrárias, v.32, n.1, p.1793-1802, 2011.

BIANCO, S.; CARVALHO, L.B.; BIANCO, M.S.; E PITELLI, R.A. Acúmulo de massa seca e de macronutrientes por plantas de Glycine max e Solanum americanum. Revista Planta Daninha, v.30, n.1, p.87-95, 2012.

BORGES, M.S.; EL-HUSNY, J.C.; CARVALHO, E.A. comportamento de cultivares e linhagens de soja transgênica em Belterra-Pará. In: SEMINÁRIO DE INICIAÇÃO CIENTÍFICA DA EMBRAPA, 16., 2012, Belém. Anais... Belém: Embrapa Amazônia Oriental, 2012. p.1-4.

BRAZ, G.B.P.; CASSOL, G.M.; ORDOÑEZ, G.A.P.; SIMON, G.A.; PROCÓPIO, S.O.; OLIVEIRA NETO, A.M.; FERREIRA FILHO, W.C.; DAN, H.A. Componentes de produção e rendimento de soja em função da época de dessecação e do manejo em pós-emergência. Revista Brasileira de Herbicidas, v.9, n.2, p.63-72, 2010. 
CAVALCANTE, A.K.; HAMAWAKI, O.T.; HAMAWAKI, R.L.; SOUZA, L.B.; NOGUEIRA, A.P.O.; HAMAWAKI, C.D.L. Adaptabilidade e estabilidade fenotípica de genótipos de soja em Porto Alegre do norte, MT. Bioscience Journal, v.30, n.4, p.942-949, 2014.

COSTA, E.D. Arranjo de plantas, características agronômicas de produtividade de soja. Botucatu: Faculdade de Ciências Agronômicas, 2013. 60p. Dissertação de Mestrado.

EMPRESA BRASILEIRA DE PESQUISA AGROPECUÁRIA - EMBRAPA. Manual de análises químicas de solos, plantas e fertilizantes. Brasília, DF: Embrapa, 2011. 627p.

EMPRESA BRASILEIRA DE PESQUISA AGROPECUÁRIA - EMBRAPA. Recomendações técnicas para a cultura da soja na Região Central do Brasil 1996/97. Londrina: Embrapa Soja, 1996. 164p. (Embrapa Soja. Sistemas de Produção).

FERREIRA, B.G.C.; FREITAS, M.M.L.; MOREIRA, G.C. Custo operacional efetivo de produção de soja em sistema de plantio direto. Revista iPecege, v.1, n.1, p.39-50, 2015.

FERREIRA, D.F. Sisvar: A Guide for its Bootstrap procedures in multiple comparisons. Ciência $e$ Agrotecnologia, Lavras-MG, v.38, n.2, p.109-112, 2014.

FREITAS, M.C.M. A cultura da soja no Brasil: o crescimento da produção brasileira e o surgimento de uma nova fronteira agrícola. Enciclopédia Biosfera, v,7, n.12, p,1-12, 2011.

MALAVOLTA, E. Manual de nutrição mineral de plantas. São Paulo: Agronômica Ceres, 2006. 638p.

MALAVOLTA, E.; VITTI, G.C.; OLIVEIRA, S.A. Avaliação do estado nutricional das plantas: princípios e aplicações. 2.ed. Piracicaba: Associação Brasileira de Potassa e do Fósforo, 1997. 319p.

MAUAD, M.; SILVA, T.L.B.; ALMEIDA NETO, A.I.; ABREU, V.G. Influência da densidade de semeadura sobre características agronômicas na cultura da soja. Revista Agrarian, Dourados-MS, v.3, n.9, p.175-181, 2010.

PELUZIO, J.M.; FIDELIS, R.R.; ALMEIDA JÚNIOR, D.; BARBOSA, V.S.; RICHTER, L.H.M.; SILVA, R.R.; AFFÉRRI, F.S. Comportamento de cultivares de soja no sul do estado do Tocantins. Bioscience Journal, Uberlândia, v.22, n.2, p.69-74, 2006.

PELUZIO, J.M.; VAZ-DE-MELO, A.; CLOMBO, G.A.; SILVA, R.; AFFÉRRI, F.S.; PIRES, L.P.M.; BARROS, H.B. Efeito da época e densidade de semeadura na produtividade de grãos de soja na Região Centro-Sul do estado do Tocantins. Revista Pesquisa Aplicada e Agrotecnologia,
V.3, n.3, p.145-153, 2010.

PERINI, L.J.; FONSECA JÚNIOR, N.S.; DESTRO, D.; PRETE, C.E.C. Componentes da produção em cultivares de soja com crescimento determinado e indeterminado. Semina: Ciências Agrárias, v.33, n.1, p.2531-2544. 2012.

PIRES, L.P.M.; PELUZIO, J.M.; CANCELLIER, L.L.; RIBEIRO, G.R.; COLOMBO, G.A; AFFÉRRI, F.S. Desempenho de genótipos de soja, cultivados na região Centro-Sul do Estado do Tocantins, safra 2009/2010. Bioscience Journal, v.28, n.2, p.214-223, 2012.

RODRIGUES, T.E.; SILVA, R.C.; SILVA, J.M.L.; OLIVEIRA, R.C., JR.; GAMA, J.R.N.F.; VALENTE, M.A. Caracterização e classificação dos solos do município de Paragominas, estado do Pará. Belém: Embrapa Amazônia Oriental, 2003. p.51.

ROCHA, R.S. Avaliação de variedades e linhagens de soja em condições de baixa latitude. Piauí: Universidade Federal do Piauí, 2009. 59p. Dissertação de Mestrado.

RÖMHELD, V.; KIRKBY, E.A. Research on potassium in agriculture: Needs and prospects. Plant Soil, v.335, p.155180, 2010.

ROSSI, R.F. Vigor de sementes, população de plantas e desempenho agronômico de soja. Botucatu: Universidade Estadual Paulista, 2012. 60p. Dissertação de Mestrado.

SACRAMENTO, P.P.; HUNGRIA, L.C.; EL-HUSNY, J.C.; FREITAS, L.S. Planting density and soybean (Glycine max L.) cultivars effects on yield components in the Amazon. Journal of Agricultural Studies, v.8, n.3, p.63-69, 2020.

SANTOS, H.P.; FONTANELI, R.S.; SPERA, S.T.; MALDANER, G.L. Rendimento de grãos de soja em diferentes sistemas de produção integração lavourapecuária. Revista Brasileira de Ciências Agrárias, v.8, n.1, p.49-56, 2013.

SEDIYAMA, T.; PEREIRA, M.G.; SEDIYAMA, C.S.; GOMES, J.L.L. Cultura da soja: I parte. 1.ed. Viçosa: Imprensa Universitária da Universidade Federal de Viçosa, 1985. 95p.

VILELA, G.F.; PEREIRA, M.J.Z.; KLEPKER, D.; MOREIRA, J.U.V.; VIEIRA, P.F.M.J.; RABELO, C.F.S.; BEZERRA, J.W.T.; PEREIRA, A.F.; SILVA NETO, S.P. Cultivares de soja: macrorregiões 4 e 5 norte e nordeste do Brasil. Londrina: Embrapa Soja, 2016. 55p.

Recebido para publicação em 10/01/2020, aprovado em 24/03/2020 e publicado em 30/04/2020. 